\title{
Bounds for maps of an interval with one reflecting critical point. I
}

by

\author{
Genadi L evin (Jerusalem)
}

\begin{abstract}
We prove real bounds for interval maps with one reflecting critical point.
\end{abstract}
In this paper we prove real bounds for maps as in the title $\left({ }^{1}\right)$. More exactly, we will consider the following class of maps.

Let $g: \bigcup I_{i} \rightarrow I$ be a map, where $I$ is an open interval around the point $c=0$ and where $I_{i}$ is a finite collection of disjoint open intervals inside the interval $I$ for which the following conditions hold:

(i) For every $i$, the map $g: I_{i} \rightarrow I$ is a homeomorphism; moreover, it is a $C^{3}$-function which extends to a smooth map on the closure of each $I_{i}$, and the Schwarzian derivative of $g, S g:=g^{\prime \prime \prime} / g^{\prime}-(3 / 2)\left(g^{\prime \prime} / g^{\prime}\right)^{2}$, is defined and negative for any $x \neq 0$.

(ii) For every $i \neq 0$, the map $g: I_{i} \rightarrow I$ is a diffeomorphism, while the map $g: I_{0} \rightarrow I$ has a unique critical point (i.e., a zero of $g^{\prime}$ ) at the point $c=0 \in I_{0}$, so that $g(x)=g(0)+(h(x))^{l}$, where $l \geq 3$ is an odd number, and $h$ is a local diffeomorphism, $h(0)=0$. We call $g: I_{0} \rightarrow I$ the central branch, and $I_{0}$ the central interval of $g$.

(ii) All iterates of the critical point $c=0$ under $g$ are in $\bigcup I_{i}$ (and, hence, well defined), $c$ is a recurrent point, and the $\omega$-limit set of the critical point $\omega(c)$ is a proper subset of $\bigcup I_{i}$, i.e., $\omega(c)$ stays away from the end points of the intervals $I_{i}$.

We always assume that $g$ has no attracting or neutral periodic orbits.

EXAmples. 1. Consider a polynomial $P$ with real coefficients and with all critical point escaping to infinity and real except for one reflecting critical

1991 Mathematics Subject Classification: 58F03, 58F23.

Research partially supported by the British Council, the Israel Ministry of Science and Arts, and the London Royal Society.

$\left({ }^{1}\right)$ For complex bounds and applications, see the second part of this paper, [LS1]. 
point. If we restrict $P$ to an interval of the real axis, it will satisfy conditions (i)-(iii) (see [LS2] for details).

2. Consider a map

$$
f(z)=\lambda z^{d} \frac{z-\frac{d+1}{d-1}}{1-\frac{d+1}{d-1} z},
$$

where $|\lambda|=1$, and $d \geq 3$. Then $f$ restricted to the circle $|z|=1$ and written in the natural coordinate of the circle satisfies conditions (i)-(ii) above (the Schwarzian is negative), with $l=3$ and with $d-1$ intervals $I_{i}$. Note that $\lambda=f(1)$ is the critical value of $f$ on the circle. Choosing $\lambda$, we can satisfy (iii) as well.

Define a sequence of first return maps

$$
g^{s_{n}}: U_{n} \rightarrow U_{n-1}
$$

inductively as follows. We set $s_{1}=1, U_{1}=I_{0}$, and $U_{0}=I$. If $U_{n-1}$ is defined, then $g^{s_{n}}: U_{n} \rightarrow U_{n-1}$ is the central branch of the first return map to $U_{n-1}$.

Let us call the map $g^{s_{n}}: U_{n} \rightarrow U_{n-1}$ central if $g^{s_{n}}(c) \in U_{n}$, and noncentral otherwise. Note that if some $g^{s_{n}}: U_{n} \rightarrow U_{n-1}$ is central, then by pulling back the central domain $U_{n}$ by the map $g^{s_{n}}$ several times, we always come to a non-central $g^{s_{m}}: U_{m} \rightarrow U_{m-1}$, where $m>n$ and where the function is the same, i.e., $s_{m}=s_{n}$.

Denote by $|U|$ the length of the interval $U$.

THEOREM 1. There exist a positive number $C$, which depends on l only, and an index $N$ so that, for every $n \geq N$, the length of every component of $U_{n-1} \backslash U_{n}$ is equal to at least $C\left|U_{n}\right|$, whenever the previous map $g^{s_{n-1}}: U_{n-1} \rightarrow U_{n-2}$ is non-central. In particular, this holds for a sequence $n_{i} \rightarrow \infty$.

REMARK 1. The corresponding real bounds for maps with one folding critical point have been proved by Martens [Ma] (for non-renormalizable maps) and by Sullivan [S] (in the renormalizable case).

REMARK 2. As in the case of a folding critical point [S], [LS2], [LS3], the real bounds of Theorem 1 are the first step towards proving the complex bounds [LS1].

REMARK 3. As in the case of a folding critical point [MS], the real bounds of Theorem 1 yield: if $\omega(c)$ is minimal, then the map is ergodic with respect to Lebesgue measure and $\omega(c)$ is of measure zero (progress in this direction for several reflecting critical points has recently been made by Edson Vargas).

Theorem 1 will be a simple consequence of Propositions 1-4 below. 
We say that, for two intervals $U \subset V$, a component of $V \backslash U$ is big compared with $U$ if the length of this component is at least $C|U|$ for some constant $C>0$ which does not depend on $U$ and $V$ ( $C$ may depend on $l$ ). We say that $U$ is well inside $V$ if both components of $V \backslash U$ are big compared with $U$.

Proposition 1. Assume that $g^{s_{n}}: U_{n} \rightarrow U_{n-1}$ is central, while the previous first return map is non-central. Let $m>n$ be minimal so that $g^{s_{m}}: U_{m} \rightarrow U_{m-1}$ is non-central and $s_{m}=s_{n}$. Then for the next map, the interval $U_{m+1}$ is well inside $U_{m}$.

Define $c_{i}:=g^{i}(c)$.

Proposition 2. Assume that all maps $g^{s_{n}}: U_{n} \rightarrow U_{n-1}$ are non-central. Let, for some $n_{0}$, the points $c_{s_{n_{0}}}$ and $c_{s_{n_{0}+1}}$ be on opposite sides of $c$. Then either $U_{n_{0}+2}$ is well inside $U_{n_{0}+1}$, or $U_{n_{0}+3}$ is well inside $U_{n_{0}+2}$.

Proposition 3. Assume that all maps $g^{s_{n}}: U_{n} \rightarrow U_{n-1}$ are non-central, and all points $c_{s_{n}}$ are on the same side of $c$ (say, always $\left.c_{s_{n}}>c\right)$. Let, for some $n_{0}$, the map $g^{s_{n_{0}}}: U_{n_{0}} \rightarrow U_{n_{0}-1}$ reverse orientation. Then $U_{n_{0}+4}$ is well inside $U_{n_{0}+3}$.

Proposition 4. Assume that all maps $g^{s_{n}}: U_{n} \rightarrow U_{n-1}$ are non-central and preserve orientation, and all points $c_{s_{n}}$ are on the same side of $c$ (say, always $\left.c_{s_{n}}>c\right)$. Then, for every $n$, the interval $U_{n}$ is well inside $U_{n-1}$.

Our proofs of Propositions 1-3 are based on combinatorial arguments, the Koebe Principle, and the Interval Adding Procedure (see below). The proof of Proposition 4 is analytic and uses a very simple geometric condition, under which the cross-ratio "does not feel" the reflecting critical point (Proposition 5). It will allow us to pass through the critical point without decreasing the cross-ratio.

As usual, if $J \subset T$ are two intervals and $L, R$ are the components of $T \backslash J$ then we define $C(T, J)$ to be the cross-ratio of this pair of intervals:

$$
C(T, J)=\frac{|J| \cdot|T|}{|L| \cdot|R|} .
$$

Cross-ratios play a crucial role in all recent results in real interval dynamics. Often, it suffices to use some qualitative estimates based on the so-called Koebe Principle. In our analysis, we shall sometimes need sharper estimates, which are based on direct use of the cross-ratio. For example, we shall often use the inequality

$$
|L| /|J| \geq C^{-1}(T, J) .
$$

Note also that $J$ is well inside $T$ if and only if $C^{-1}(T, J)$ is greater than a positive constant (which does not depend on the intervals). 
If $f$ is a map which is a diffeomorphism of $T$ onto its image and $S f<0$ then (see $[\mathrm{MS}]$ )

$$
C^{-1}(T, J) \geq C^{-1}(f(T), f(J)) .
$$

In our case we shall apply this to maps $f$ of the form $g^{n}$. Since $S g<0$ one also has $S g^{n}<0$ so the previous inequality applies when we take $f=g^{n}$ and $g^{n} \mid T$ is a diffeomorphism. We will use the following amusing extension of this classical fact:

Proposition $5\left({ }^{2}\right)$. Let $L$ be the left component of $T \backslash J$, so that if we write $L=\left(x_{1}, x_{2}\right)$, then $x_{1}<0<x_{2}$, and, moreover, $x_{2}>\left|x_{1}\right|$. Fix a real number $l>1$, and introduce a map

$$
F(x)=\frac{x}{|x|} \cdot|x|^{l}, \quad x \neq 0, \quad F(0)=0 .
$$

Then

$$
C^{-1}(T, J) \geq C^{-1}(F(T), F(J)) .
$$

Proof. First of all, we can assume that $x_{1}=-1$, and then $x_{2}=1+\delta<$ $x_{3}<x_{4}$, where $R=\left(x_{3}, x_{4}\right)$ and $\delta>0$. To calculate the cross-ratios, we will use the following well known equality:

$$
\ln \frac{1+C^{-1}(F(T), F(J))}{1+C^{-1}(T, J)}=\int_{x_{3}}^{x_{4}} d y \int_{x_{1}}^{x_{2}} U(x, y) d x
$$

where

$$
U(x, y)=\frac{F^{\prime}(x) F^{\prime}(y)}{(F(x)-F(y))^{2}}-\frac{1}{(x-y)^{2}} .
$$

Fix $y \in\left(x_{3}, x_{4}\right)$, and write the integral over $x$ as a sum of two integrals $I_{1}$ and $I_{2}$, where $x$ in $I_{1}$ varies from -1 to 1 , and in $I_{2}$ from 1 to $1+\delta$. Then $I_{2}<0$, since the function $x^{l}, x>0$, has negative Schwarzian derivative. For the first integral, we have

$$
I_{1}=\int_{-1}^{0} U(x, y) d x+\int_{0}^{1} U(x, y) d x=\int_{0}^{1} V(x, y) d x
$$

where

$$
V(x, y)=\frac{l^{2} x^{l-1} y^{l-1}}{\left(x^{l}+y^{l}\right)^{2}}+\frac{l^{2} x^{l-1} y^{l-1}}{\left(x^{l}-y^{l}\right)^{2}}-\frac{1}{(x+y)^{2}}-\frac{1}{(x-y)^{2}} .
$$

After computing the integral, we obtain

$$
I_{1}=\frac{2 l}{y}\left\{\frac{l a^{l-1}}{1-a^{2 l}}-\frac{1}{1-a^{2}}\right\}
$$

$\left({ }^{2}\right)$ This statement was established during the author's visit to Sebastian van Strien at Warwick and numerous conversations with him. 
where the parameter $a=1 / y \in(0,1)$. Since the expression in $\{\ldots\}$ is negative, we are done.

We make use of two principles.

Koebe PRINCIPLE [MS]. Let $T$ be an interval from a component of the domain of definition of some iterate $g^{s}$, and so that $T=L \cup J \cup R$, where: (a) $g^{s} \mid J$ has a unique critical point at c, and (b) $g^{s} \mid L$ and $g^{s} \mid R$ have at most $N$ critical points. Let $K>0$ be so that

$$
\left|g^{s}(L)\right| /\left|g^{s}(J)\right|,\left|g^{s}(R)\right| /\left|g^{s}(J)\right| \geq K \text {. }
$$

Then

$$
|L| /|J|,|R| /|J| \geq C,
$$

where $C>0$ depends on $K, N$, and on the map $g$. Moreover, given $g$, there exists $\varepsilon>0$ so that if $\left|g^{i}(T)\right| \leq \varepsilon$ for $i=0,1, \ldots, s$, then the constant $C$ depends merely on $K$ and $N$.

For any two intervals $U, V$, denote by $[U, V]$ the minimal interval which contains $U$ and $V$.

Interval Adding Procedure. Given $g^{s_{n}}: U_{n} \rightarrow U_{n-1}$, let $t_{n}^{+}$be a maximal interval outside $U_{n}$ and to the right of $U_{n}$ with a common boundary point with $U_{n}$, so that $g^{s_{n}} \mid t_{n}^{+}$is defined and a diffeomorphism. The interval $t_{n}^{-}$is defined in the same way, but to the left of $U_{n}$.

Let $t$ be either $t_{n}^{+}$or $t_{n}^{-}$. Note that one end point of $g^{s_{n}}(t)$ is in $\partial U_{n-1}$. If the other end point of $g^{s_{n}}(t)$ is an end point of the range $I$ of the map $g$, then we set $k=0$ and stop. Otherwise the boundary point of $t$ which is different from $\partial U_{n}$ is a critical point of $g^{s_{n}}$ : there exists $i, 1 \leq i \leq s_{n}-1$, so that $c$ lies at the boundary of the interval $g^{i}(t)$. In this case we will make an operation called "adding the interval $U_{n-1}$ ":

Since $g^{i}\left(U_{n}\right)$ is outside $U_{n-1}$ and $g^{i}(t)$ has $c$ in its boundary, the interval $g^{i}(t)$ also contains a boundary point of $U_{n-1}$. Hence, either $i+s_{n-1}>$ $s_{n}$, or $g^{i+s_{n-1}}(t)$ contains a boundary point of $U_{n-2}$. Hence, again either $i+s_{n-1}+s_{n-2}>s_{n}$, or $g^{i+s_{n-1}+s_{n-2}}(t)$ contains a boundary point of $U_{n-3}$. We continue this process until we find $k \geq 1$ so that

$$
S:=i+s_{n-1}+\ldots+s_{n-k+1}<s_{n},
$$

while

$$
S+s_{n-k} \geq s_{n} .
$$

Then $g^{S}(t)$ contains a boundary point of $U_{n-k}$. Let us extend the interval $g^{S}(t)$ so that it turns into the interval $\left[U_{n-1}, g^{S}(t)\right]$, and call this operation "adding the interval $U_{n-1}$ ".

The boundary point of $t$ different from $\partial U_{n}$ lies in a component of $U_{m-1} \backslash$ $U_{m}$ for some $m \leq n$. Since the points of $\partial U_{m-1}$ are nice, there exists a unique 
extension $\widehat{t}$ of $t$ inside $U_{m-1} \backslash U_{m}$ so that

$$
g^{S}(\widehat{t})=\left[U_{n-1}, g^{S}(t)\right] .
$$

Lemma 1. (i) $g^{s_{n}}(\widehat{t})$ covers an interval $g^{j}\left(U_{n-1}\right)$ for some $1 \leq j \leq$ $s_{n-1}-1$.

(ii) The map $g^{s_{n}} \mid \widehat{t}$ has at most $k$ critical points.

(iii) Let $\widehat{t}_{n}^{ \pm}$be the intervals corresponding to $t_{n}^{ \pm}$, and $k_{n}^{ \pm}$be the corresponding numbers. If $k_{n}^{ \pm} \leq N$, then $\left|t_{n}^{ \pm}\right| \geq C\left|U_{n}\right|$, where $C>0$ only depends on $l$ and $N$.

P r o of. Since $g^{S}(\widehat{t})$ contains $U_{n-1}$ and $S+s_{n-k} \geq s_{n}$, the interval $g^{s_{n}}(\widehat{t})$ contains some $g^{j}\left(U_{n-1}\right)$, where $j \leq s_{n-k} \leq s_{n-1}-1$. To prove the second statement, first note that the map $g^{i} \mid \widehat{t}$ has no critical points, since $g^{i}(\widehat{t} \backslash t) \subset$ $U_{n-1}$ and $i<s_{n}$. On the other hand, the interval $g^{i}(\widehat{t})$ covers $c$. Hence, the map $g^{s_{n-1}} \mid g^{i}(\widehat{t})$ has one critical point. Since $g^{s_{n-1}}\left(g^{i}(\widehat{t})\right) \backslash g^{s_{n-1}}\left(g^{i}(t)\right)$ is part of $U_{n-2}$, the map $g^{s_{n-2}} \mid g^{i+s_{n-1}}(\widehat{t})$ can have at most one critical point, and so on until $g^{S}(\widehat{t})$ (each time at most one critical point can be added). The iterate of $g$ from the latter interval to the interval $g^{s_{n}}(\widehat{t})$ does not have critical points, by definition of $k$. The last statement follows from the previous ones and from the Koebe Principle (here we use the smallest interval argument).

REMARK 4. In what follows, if $g^{s_{n}}\left(\widehat{t}_{n}^{ \pm}\right)$covers more than one interval $g^{j}\left(U_{n-1}\right)$, we shorten $\widehat{t}_{n}^{ \pm}$so that $g^{s_{n}}\left(\widehat{t}_{n}^{ \pm}\right)$covers exactly one interval of this form.

Let us call the numbers $k_{n}^{ \pm}$the parameters, or the numbers of steps of the procedure.

To start with, let us prove the following

LEMMA 2. (i) Let $^{j_{1}}\left(U_{n}\right)$ and $g^{j_{2}}\left(U_{n}\right)$ be two neighbours of $U_{n}$ from the collection $g^{j}\left(U_{n}\right), j=1, \ldots, s_{n}-1$. Then $U_{n}$ is well inside $\left[g^{j_{1}}\left(U_{n}\right), g^{j_{2}}\left(U_{n}\right)\right]$.

(ii) Let the map $g^{s_{n-1}}: U_{n-1} \rightarrow U_{n-2}$ be non-central. Then, at least for one component $K$ of $U_{n-1} \backslash U_{n}$, the iterate $g^{s_{n}}(K)$ covers $g^{j}\left(U_{n}\right)$ for some $1 \leq j \leq s_{n}-1$. If , for example, $c_{s_{n-1}}>c$, then $K$ is the left (resp. right) component of $U_{n-1} \backslash U_{n}$ depending on whether $g^{s_{n-1}}: U_{n-1} \rightarrow U_{n-2}$ preserves (resp. reverses) orientation.

(iii) Let the map $g^{s_{n-1}}: U_{n-1} \rightarrow U_{n-2}$ be non-central. Then one of the two components of $U_{n-1} \backslash U_{n}$ is big compared with $U_{n}$.

Proof. (i) follows from the shortest interval argument and from the Koebe Principle.

(ii) Let $c_{s_{n-1}}>c$. Then the end points of $g^{s_{n-1}}(K)$ already lie on opposite sides of the critical point $c$. 
(iii) Denote by $t$ the interval around $U_{n}$ which is mapped by $g^{s_{n}}$ onto $\left[g^{j_{1}}\left(U_{n}\right), g^{j_{2}}\left(U_{n}\right)\right]$. The map $g^{s_{n}} \mid t$ has at most 3 critical points, because $\left[g^{j_{1}}\left(U_{n}\right), g^{j_{2}}\left(U_{n}\right)\right]$ contains precisely 3 critical values of this map. Since $g^{s_{n-1}}: U_{n-1} \rightarrow U_{n-2}$ is non-central, by (ii) at least one of the ends of $t$ (say, the left one) lies inside $U_{n-1} \backslash U_{n}$. Assume now the contrary: every component of $U_{n-1} \backslash U_{n}$ is not big compared with $U_{n}$. Then, by (i) the interval $U_{n-1}$ is well inside $\left[g^{j_{1}}\left(U_{n}\right), g^{j_{2}}\left(U_{n}\right)\right]$. Pulling $\left[g^{j_{1}}\left(U_{n}\right), g^{j_{2}}\left(U_{n}\right)\right]$ back by $g^{s_{n}}$ to $t$ (so that $U_{n-1}$ is pulled back to $U_{n}$ ) we then conclude that the left component of $U_{n-1} \backslash U_{n}$ is big compared with $U_{n}$. A contradiction.

LEMmA 3. Let the map $g^{s_{n-1}}: U_{n-1} \rightarrow U_{n-2}$ be non-central, and let $t$ be an interval around $U_{n}$ such that $g^{s_{n}}(t)=\left[g^{j_{1}}\left(U_{n}\right), g^{j_{2}}\left(U_{n}\right)\right]$ (where, as in Lemma $2, g^{j_{1}}\left(U_{n}\right)$ and $g^{j_{2}}\left(U_{n}\right)$ are two neighbours of $U_{n}$ from the collection $\left.g^{j}\left(U_{n}\right), j=1, \ldots, s_{n}-1\right)$. Then $t \subset U_{n-2}$. In particular, $t_{n+1}^{ \pm} \subset U_{n-2} \backslash U_{n+1}$ (see Remark 4).

Proof. First, one can assume without loss of generality that $c_{s_{n-1}}>c$. Now, the map $g^{s_{n-1}}: U_{n-1} \rightarrow U_{n-2}$ is non-central, and hence $g^{s_{n-1}}\left(U_{n}\right)$ lies in the right component of $U_{n-2} \backslash U_{n-1}$. It follows immediately that the right end of $t$ belongs to $U_{n-2}$. To prove the same for the left end of $t$ one should consider different combinations of the following possibilities: $g^{s_{n-1}}: U_{n-1} \rightarrow U_{n-2}$ preserves or reverses orientation, $c_{s_{n-1}}$ and $c_{s_{n-2}}$ are on the same side of $c$, or on the opposite sides:

1) $g^{s_{n-1}}: U_{n-1} \rightarrow U_{n-2}$ preserves orientation. Then the component $K$ defined in Lemma 2(ii) is the left component of $U_{n-1} \backslash U_{n}$, and hence the left end of $t$ is inside $K$, and we are done in this case.

2) $g^{s_{n-1}}: U_{n-1} \rightarrow U_{n-2}$ reverses orientation. Consider also the map $g^{s_{n-2}}: U_{n-2} \rightarrow U_{n-3}$.

2a) Let $s_{n-2}=s_{n-1}$ (i.e., the return to $U_{n-3}$ is central). Denote by $r(V \backslash U)$ (resp. $l(V \backslash U))$ the right (left) component of $V \backslash U$. Since we are in case 2, we have $g^{s_{n-1}}\left(r\left(U_{n-2} \backslash U_{n-1}\right)\right)=l\left(U_{n-3} \backslash U_{n-2}\right)$. But $g^{s_{n-1}}\left(U_{n}\right) \subset$ $r\left(U_{n-2} \backslash U_{n-1}\right)$. Hence, $g^{2 s_{n-1}}\left(U_{n}\right) \subset l\left(U_{n-3} \backslash U_{n-2}\right)$. This is enough, since $g^{s_{n}}$ takes the end points of $U_{n-2}$ outside $U_{n-3}$.

$2 \mathrm{~b})$ Let $s_{n-2}<s_{n-1}$ (i.e., the return to $U_{n-3}$ is non-central). If $c_{s_{n-2}}<c$, then $g^{s_{n-2}}\left(U_{n}\right) \subset l\left(U_{n-3} \backslash U_{n-2}\right)$, and because $g^{s_{n}}$ takes the end points of $U_{n-2}$ outside $U_{n-3}$, we are done. Let $c_{s_{n-2}}>c$. Then $g^{s_{n-2}}\left(U_{n}\right) \subset r\left(U_{n-3} \backslash\right.$ $\left.U_{n-2}\right)$. Hence, already $g^{s_{n-1}}\left(l\left(U_{n-2} \backslash U_{n}\right)\right)$ covers $g^{s_{n-2}}\left(U_{n}\right)$ (we are in case $2)$. Thus all cases have been considered.

Proof of Proposition 1. The idea of the proof is to use the space around $U_{n}$ given by the first part of Lemma 2 and pull it back by $g^{s_{n}}$. Since the return is central, we again obtain a map from our sequence of the first return maps. 
First, for the interval $t$ from Lemma 3, we have $t \subset U_{n-2}$. It follows immediately from this observation and from Lemma $2(\mathrm{i})$ that $U_{n+1}$ is well inside $U_{n-2}$ (here we use the fact that $s_{n+1}=s_{n}$ ). Consider now two cases:

1. $m-n \geq 2$. Then we can pull $U_{n+1}$ back by the same central branch one more time and find that $U_{n+2}$ (and hence $U_{m}$ ) is well inside $U_{n-1}$. Since $g^{s_{n}}: U_{m} \rightarrow U_{m-1}$ is non-central, the next map, i.e., the first return to $U_{m}$ will already have a bound which is determined by the space between $U_{m}$ and $U_{n-1}$. Therefore, $U_{m+1}$ will be well inside $U_{m}$.

2. $m-n=1$. We apply in this case the Interval Adding Procedure to the map $g^{s_{m+1}}: U_{m+1} \rightarrow U_{m}$. Then either we finish this procedure after two steps, or we cover a component of $U_{n-2} \backslash U_{n+1}$, which, as we know, is big compared with $U_{n+1}$. In both cases, we again conclude that $U_{m}$ is well inside $U_{n-1}$. (Note that $g^{s_{m+1}}\left(\partial U_{m}\right)$ is outside $U_{n-2}$.) Therefore, $U_{m+1}$ is well inside $U_{m}$.

Proof of Proposition 2. According to Lemma 2, one of the two components of $U_{n-1} \backslash U_{n}$ is big compared with $U_{n}$.

One can assume that $c_{s_{n_{0}}}<c$ and $c_{s_{n_{0}+1}}>c$. Note that the interval $g^{s_{n_{0}}}\left(U_{n_{0}+1}\right)$ is in $U_{n_{0}-1} \backslash U_{n_{0}}$. Therefore, by Lemma 2(i), the left component of $U_{n_{0}-1} \backslash U_{n_{0}+1}$ is big compared with $U_{n_{0}+1}$. If we now assume that the right component of $U_{n_{0}-1} \backslash U_{n_{0}}$ is big (compared with $U_{n_{0}}$ ), then we see that $U_{n_{0}+2}$ is well inside $U_{n_{0}+1}$ (we apply the Interval Adding Procedure to the map from $U_{n_{0}+2}$ to $U_{n_{0}+1}$, and either we finish it with $k \leq 2$ and then we "add the interval" or otherwise without adding we cover a component of $\left.U_{n_{0}-1} \backslash U_{n_{0}}\right)$. Therefore, we should assume that the left component of $U_{n_{0}-1} \backslash U_{n_{0}}$ is big (compared with $U_{n_{0}}$ ). By the same reason, we should assume that the right component of $U_{n_{0}} \backslash U_{n_{0}+1}$ (which contains $c_{s_{n_{0}+1}}$ ) is also big compared with $U_{n_{0}+1}$ (otherwise $U_{n_{0}+3}$ would be well inside $U_{n_{0}+2}$ ). But then again $U_{n_{0}+2}$ is well inside $U_{n_{0}+1}$.

Proof of Proposition 3. Note that the interval $g^{s_{n_{0}+1}}\left(U_{n_{0}+2}\right)$ is in the right component of $U_{n_{0}} \backslash U_{n_{0}+1}$.

Let us first show that either

- $s_{n_{0}}+s_{n_{0}+1}<s_{n_{0}+2}$ and the interval $g^{s_{n_{0}}+s_{n_{0}+1}}\left(U_{n_{0}+2}\right)$ is in the left component of $U_{n_{0}-1} \backslash U_{n_{0}}$, or

- $U_{n_{0}+4}$ is well inside $U_{n_{0}+3}$.

Namely, consider the Interval Adding Procedure for the map $g^{s_{n_{0}+2}}$ : $U_{n_{0}+2} \rightarrow U_{n_{0}+1}$, and let $t$ be one of the two intervals there. Then either (a) we finish the procedure after two steps, or (b) $g^{i+s_{n_{0}+1}}(t)$ has $c_{s_{n_{0}+1}}$ as an end point, and contains an end point of $U_{n_{0}}$, and, moreover, $g^{s_{n_{0}}+1}$ is a diffeomorphism of this interval. In case (b), the interval $g^{s_{n_{0}}+s_{n_{0}+1}}\left(U_{n_{0}+2}\right)$ is to the left of $c$. Indeed, the map $g^{s_{n_{0}}}$ takes the right end of $U_{n_{0}}$ to the left 
end of $U_{n_{0}-1}$ (we use the fact that $g^{s_{n_{0}}}: U_{n_{0}} \rightarrow U_{n_{0}-1}$ reverses orientation). Hence, in case (b), this map takes $c_{s_{n_{0}+1}}$ to the left of $c$ as well. So, at least part of the interval $g^{s_{n}+s_{n_{0}+1}}\left(U_{n_{0}+2}\right)$ is to the left of $c$. If the latter interval covered $c$ then $s_{n_{0}}+s_{n_{0}+1}=s_{n_{0}+2}$ and since all $c_{s_{n}}$ are to the right, $g^{s_{n}}\left(g^{i+s_{n_{0}+1}}(t)\right)$ would cover $c$.

Thus, either $g^{s_{n_{0}}+s_{n_{0}+1}}\left(U_{n_{0}+2}\right)$ is to the left of $c$, or the parameter for each side of the procedure is $\leq 2$. In the latter case, we use Lemmas 1 and 3 to conclude that $U_{n_{0}+3}$ is well inside $U_{n_{0}+1}$, which implies that $U_{n_{0}+4}$ is well inside $U_{n_{0}+3}$.

Finally, we can assume that $g^{s_{n_{0}+1}}\left(U_{n_{0}+2}\right)$ and $g^{s_{n_{0}}+s_{n_{0}+1}}\left(U_{n_{0}+2}\right)$ are on the opposite sides of $c$ (but inside $U_{n_{0}-1}$ ). Then we apply the procedure to the map $g^{s_{n_{0}+3}}: U_{n_{0}+3} \rightarrow U_{n_{0}+2}$ this time, and either we finish it after 3 steps, or we cover one of these two intervals. Note that $g^{s_{n_{0}+3}}\left(\partial U_{n_{0}+1}\right)$ is outside $U_{n_{0}-1}$. In the case considered, one can assume that $\widehat{t}_{n_{0}+3}^{ \pm} \subset U_{n_{0}+1} \backslash$ $U_{n_{0}+3}$. Therefore, in any case, $U_{n_{0}+3}$ is well inside $U_{n_{0}+1}$, and then $U_{n_{0}+4}$ is well inside $U_{n_{0}+3}$ (the returns are non-central).

In order to prove Proposition 4, we first prove two lemmas. Set $U_{n}=$ $\left(-l_{n}, r_{n}\right)$ and $\varrho_{n}=l_{n} / r_{n}$, and define

$$
\delta_{n}^{+}=\frac{r_{n-1}-r_{n}}{r_{n}+l_{n}}, \quad \delta_{n}^{-}=\frac{l_{n-1}-l_{n}}{r_{n}+l_{n}} .
$$

By the assumption, $c_{s_{n}} \in\left(r_{n}, r_{n-1}\right)$ for any $n$.

The next lemma is a direct consequence of Proposition 5 from the beginning of the paper.

Lemma 4. Under the conditions of Proposition 4, assume that

$$
r_{n}>l_{n-1}
$$

for all $n$. Then $C^{-1}\left(U_{n-1}, U_{n}\right)$ tends to infinity as $n$ tends to infinity.

Proof. Fix $n$, and look at the map $g^{s_{n-1}}: U_{n-1} \rightarrow U_{n-2}$. We have

$$
C^{-1}\left(g\left(U_{n-1}\right), g\left(U_{n}\right)\right) \geq C^{-1}\left(U_{n-2}, g^{s_{n-1}}\left(U_{n}\right)\right) .
$$

Since the return is non-central,

$$
g^{s_{n-1}}\left(U_{n}\right) \subset\left(r_{n-1}, r_{n-2}\right) .
$$

By condition (1), $r_{n-1}>l_{n-2}$, so that we can apply Proposition 5 :

$$
C^{-1}\left(U_{n-2}, g^{s_{n-1}}\left(U_{n}\right)\right) \geq C^{-1}\left(g\left(U_{n-2}\right), g^{s_{n-1}+1}\left(U_{n}\right)\right) .
$$

Now we look at the map $g^{s_{n-2}}: U_{n-2} \rightarrow U_{n-3}$, and since the return is again non-central and on the same (right) side, we can continue, up to the level $U_{0}=I$ :

$$
C^{-1}\left(g\left(U_{n-1}\right), g\left(U_{n}\right)\right) \geq C^{-1}\left(I, g^{S_{n}}\left(U_{n}\right)\right),
$$


where $S_{n}=s_{n-1}+\ldots+s_{1}$. On the other hand,

$$
\left|g^{S_{n}}\left(U_{n}\right)\right| \rightarrow 0
$$

as $n \rightarrow \infty$ (because any two intervals $g^{i}\left(U_{n}\right), g^{j}\left(U_{m}\right)$ are either disjoint or one contains the other and because no wandering interval exists [MS]), and this interval is away from the end points of $I$ (condition (iii) of the definition of the class of maps $g)$. Therefore, $C^{-1}\left(I, g^{S_{n}}\left(U_{n}\right)\right) \rightarrow \infty$.

REMARK 5. We have also proved the following: if condition (1) holds for all $n$ between some $n_{0}$ and $n_{1}>n_{0}$, then

$$
C^{-1}\left(g\left(U_{n_{1}}\right), g\left(U_{n_{1}+1}\right)\right) \geq C_{n_{0}-1}^{-1},
$$

where $C_{n_{0}-1}^{-1}$ is the infimum of the cross-ratios $C^{-1}\left(U_{n_{0}-1}, K\right)$ over all those components $K$ of the first return map to $U_{n_{0}-1}$ which contain an iterate of the critical point.

Lemma 5. Under the conditions of Proposition 5, assume that

$$
r_{m-1}(1+\varepsilon) \leq l_{m-2}
$$

for some $m$ and some small enough $\varepsilon>0$. Then $U_{m+1}$ is well inside $U_{m}$.

Pro of. For any $n,(\mathrm{a}),(\mathrm{b})$, and (c) below hold:

(a) By Lemma 2(i), there exists a positive constant $\delta_{0}$, which depends only on $l$, such that each of the two gaps between $U_{n}$ and the end points of the interval $\left[U_{n}^{\prime}, U_{n}^{\prime \prime}\right]$ is at least $\delta\left|U_{n}\right|$, where $U_{n}^{\prime}, U_{n}^{\prime \prime}$ are the neighbours of $U_{n}$ from the collection $g^{j}\left(U_{n}\right), j=1, \ldots, s_{n}-1$.

(b) Since the returns are non-central and to the right of 0 , the interval $g^{s_{n-2}}\left(U_{n-1}\right)$ lies in $\left(r_{n-2}, r_{n-3}\right)$, and $g^{s_{n}}\left(\left(r_{n}, r_{n-1}\right)\right)$ covers $\left(r_{n-1}, r_{n-3}\right)$. Hence, by the Interval Adding Procedure and Lemma 1, there exists an interval $\hat{t}_{n}^{+}=\left(r_{n}, x_{n}\right)$, where $x_{n} \in\left(r_{n}, r_{n-1}\right)$, such that $\left.g^{s_{n}}\right|_{\hat{t}_{n}^{+}}$has at most one critical point and $g^{s_{n}}\left(\widehat{t}_{n}^{+}\right)$covers some interval of the form $g^{j}\left(U_{n-1}\right)$, $j=1, \ldots, s_{n-1}-1$. In particular, by (a), $\left|g^{s_{n}}\left(\widehat{t}_{n}^{+}\right)\right| \geq \delta_{0}\left|U_{n-1}\right|$.

(c) Since the returns are non-central, and to the right of 0 , there exists an interval $\widehat{t}_{n}^{-}=\left(-y_{n},-l_{n}\right)$, where $-y_{n} \in\left(-l_{n-1},-l_{n}\right)$, such that $\left.g^{s_{n}}\right|_{\hat{t}_{n}^{-}}$ has at most one critical point and $g^{s_{n}}\left(\widehat{t}_{n}^{-}\right)$covers some interval of the form $g^{j}\left(U_{n}\right), j=1, \ldots, s_{n}-1$. In particular, by (a), $\left|g^{s_{n}}\left(\widehat{t}_{n}^{-}\right)\right| \geq \delta_{0}\left|U_{n}\right|$. (Note the difference between (b) and (c)). Moreover, by the same reason, there exists an interval $t \subset \widehat{t}_{n}^{-}$such that the map $g^{s_{n}}: t \rightarrow\left(-l_{n-2},-l_{n-1}\right)$ is one-to-one.

Consider now two cases:

1. $\delta_{m-1}^{-} \geq \delta_{0} / 10$. Then, by (b) and (c) (with $n=m$ ), $U_{m}$ is well inside $U_{m-1}$, and, hence, $U_{m+1}$ is well inside $U_{m}$.

2. $\delta_{m-1}^{-} \leq \delta_{0} / 10$. Then

$$
l_{m-2} \leq l_{m-1}+\frac{\delta_{0}}{10}\left(l_{m-1}+r_{m-1}\right) .
$$


Together with $l_{m-2} \geq r_{m-1}(1+\varepsilon)$, this gives

$$
\varrho_{m-1} \geq \frac{10(1+\varepsilon)-\delta_{0}}{10(1+\varepsilon)+\delta_{0}}
$$

Consider 3 subcases:

2.1. $\delta_{m}^{-} \geq \delta_{0} / 10$. Then $U_{m+1}$ is well inside $U_{m}$ (see case 1 ).

2.2. $\delta_{m}^{-} \leq \delta_{0} / 10$ and $\delta_{m}^{+} \leq 10$. Consider the two gaps between $U_{m-1}$ and the end points of $g^{s_{m}}\left(\widehat{t}_{m}^{-} \cup U_{m} \cup \widehat{t}_{m}^{+}\right)$. The right gap is at least $\delta_{0}\left|U_{m-1}\right|$. The left gap is at least $9 \delta_{0}\left|U_{m}\right| / 10$. But

$$
\frac{9 \delta_{0}\left|U_{m}\right| / 10}{\left|U_{m-1}\right|}=\frac{9 \delta_{0} / 10}{1+\left(\delta_{m}^{-}+\delta_{m}^{+}\right)} \geq \frac{9 \delta_{0} / 10}{1+\delta_{0} / 10+10}=: \delta_{1}>0,
$$

and therefore $U_{m}$ is well inside $U_{m-1}$.

2.3. $\delta_{m}^{-} \leq \delta_{0} / 10$ and $\delta_{m}^{+} \geq 10$. Then

$$
\varrho_{m-1}=\frac{l_{m-1}}{r_{m-1}}=\frac{l_{m}+\delta_{m}^{-}\left(l_{m}+r_{m}\right)}{r_{m}+\delta_{m}^{+}\left(l_{m}+r_{m}\right)} \leq \frac{1+\delta_{m}^{-}}{\delta_{m}^{+}} \leq \frac{1}{10}\left(1+\frac{\delta_{0}}{10}\right) .
$$

This contradicts (3) if $\varepsilon$ and $\delta_{0}$ are small enough (but fixed).

Since all the cases have been considered, the statement is proved.

Proof of Proposition 4. This is a simple application of Lemmas 4-5 above. Indeed, if the conditions of Lemma 4 are satisfied, we are done. Otherwise, there are $m$ and $\varepsilon>0$ small enough so that (2) holds. By Lemma $5, U_{m+1}$ is then well inside $U_{m}$, and therefore each component of the first return map to $U_{m+1}$ is well inside $U_{m+1}$. In particular, the quantity $C_{m+1}^{-1}$ which was defined in the remark after the proof of Lemma 4 is bounded away from zero by a constant which depends on $l$ only. Now, if (1) holds for $n=m+2, m+3, m+4$, then $U_{m+6}$ is well inside $U_{m+5}$ by the remark after the proof of Lemma 4 , and we start over again replacing $m$ by $m+5$. If not, then we apply Lemma 5 to $m+2, m+3$, or $m+4$ instead of $m-1$, and start again as well.

Proof of Theorem 1. We repeat the proof of Proposition 4, replacing Lemma 5 by one of Propositions 1-3 if needed.

REMARK 6. Combining the proofs of the paper with some methods of the recent thesis by O. Kozlovski, one can get rid of the negative Schwarzian derivative condition to obtain the same real bounds for any $C^{2}$-map with one reflecting critical point (see [LS1] for details).

Acknowledgments. The author thanks C. Petersen, S. van Strien, and J.-C. Yoccoz for helpful conversations, and the referee for his careful reading of the paper and for useful comments. Furthermore, the author thanks the Mathematics Institute at Warwick, where part of this work was done, for its hospitality. 


\section{References}

[LS1] G. Levin and S. van Strien, Bounds for maps of an interval with one reflecting critical point. II, in preparation.

[LS2] - - - Total disconnectedness of the Julia set of real polynomials, preprint IHES, 1996/68 (1996).

[LS3] - - - Local connectivity of the Julia set of real polynomials, Ann. of Math., to appear.

[Ma] M. Martens, Interval dynamics, thesis, Delft, 1990.

[MS] V. de Melo and S. van Strien, One-Dimensional Dynamics, Ergeb. Math. Grenzgeb. 25, Springer, 1993.

[S] D. Sullivan, Bounds, quadratic differentials, and renormalization conjectures, in: AMS Centennial Publications, Vol. II, Amer. Math. Soc., 1992, 417-466.

Institute of Mathematics

Hebrew University

Givat Ram 91904

Jerusalem, Israel

E-mail: levin@math.huji.ac.il

Received 10 November 1997;

in revised form 30 November 1997 